Supporting Information for

\title{
Expression of human DNA helicase B is affected by G-quadruplexes in the promoter
}

Maroof Khan Zafar ${ }^{1}$, Lindsey Hazeslipp, Muhammad Zain Chauhann', and Alicia K. Byrd ${ }^{1,2, *}$

${ }^{1}$ Department of Biochemistry and Molecular Biology, University of Arkansas for Medical Sciences, Little Rock, Arkansas, 72205, USA

${ }^{2}$ Winthrop P. Rockefeller Cancer Institute, Little Rock, Arkansas, 72205, USA 
Table S1. Oligonucleotide Sequences

\begin{tabular}{|c|c|}
\hline Name & Sequence (5'-to-3') \\
\hline HELB-G4-1 & CGAGGGGAGGGCATGTGGAAGGGGCGGGGCAA \\
\hline HELB-G4-2 & GGCGGGTTAGGGGCGGGGAAGGGAGA \\
\hline HELB-G4-3 & CGAGGGGCT $\underline{G G A G A T G G G C G G G G C T T}$ \\
\hline HELB-G4-123 & $\begin{array}{l}\text { CGAGGGGAGGGCATGTGGAAGGGGCGGGGCAAGGCGGGTTAGGGGCGGGGAAGGGAGAGCG } \\
\text { AGGGGCTGGGAGATGGGCGGGGCTT }\end{array}$ \\
\hline HELB-iM-1 & TTGCCCCGCCCCTTCCACATGCCCTCCCCTCG \\
\hline HELB-iM-2 & TCTCCCTTCCCCGCCCCTAACCCGCC \\
\hline HELB-iM-3 & AAGCCCCGCCCATCTCCCAGCCCCTCG \\
\hline HELB-iM-123 & $\begin{array}{l}\text { AAGCCCCGCCCATCTCCCAGCCCCTCGCTCTCCCTTCCCCGCCCCTAACCCGCCTTGCCCC } \\
\text { GCC }\end{array}$ \\
\hline $\begin{array}{l}\text { 5'F-HELB-G4- } \\
\text { 123-3'bio }\end{array}$ & 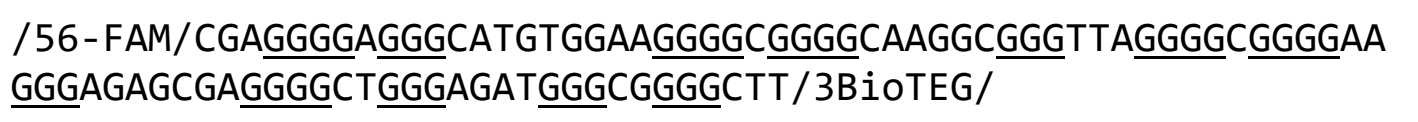 \\
\hline HELB-G4-mutG & $\begin{array}{l}\text { CGAaaaaAaaaCATGTGaAAaaaaCaaaaCAAGGCaaaTTAaaaaCaaaaAAaaaAGAGCG } \\
\text { AaaaaCTaaaAGATaaaCaaaaCTT }\end{array}$ \\
\hline $\begin{array}{l}\text { HELB-G4-mut- } \\
\text { prot }\end{array}$ & $\begin{array}{l}\text { CGAGaaaAaaaCATGTGGAAGGGGCGaaaCAAGGCGGGTTAGGGGCGaaaAAaaaAGAGCG } \\
\text { AGaaaCTGGGAGATaaaCGaaaCTT }\end{array}$ \\
\hline $\begin{array}{l}\text { HELB-G4-mut- } \\
\text { react }\end{array}$ & $\begin{array}{l}\text { CGAGGGGAGGGCATGTGGAAaaaaCGGGGCAAGGCaaaTTAaaaaCGGGGAAGGGAGAGCG } \\
\text { A } \underline{\underline{G G G G C T}} \text { aaaAGAT } \underline{\underline{G G G C G G G G C T T}}\end{array}$ \\
\hline 5'F-primer & /56-FAM/CGCCAGAGTCTTCTCAGTCACGACC \\
\hline $\begin{array}{l}\text { HELB G4 } \\
\text { template }\end{array}$ & 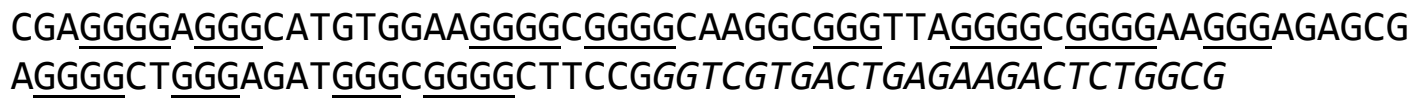 \\
\hline
\end{tabular}

Potential $\mathrm{G} 4$ and $\mathrm{iM}$ forming regions are underlined.

Duplex forming regions are italicized.

Bases which are mutated are bold. 
Table S2. Primer Sequences

\begin{tabular}{lll}
\hline Gene & Primer & Sequence (5'-to-3') \\
\hline HELB & HELB For & AAGTGGGAAGGTGATGTG \\
& HELB Rev & CTGAAGCATTCTGGTCTTGTTC \\
MYC & MYC For & TTCGGGTAGTGGAAACCAG \\
& MYC Rev & AGTAGAAATACGGCTGCACC \\
ACTB & ACTB For & ACCTTCTACAATGAGCTGCG \\
& ACTB Rev & CCTGGATAGCAACGTACATGG \\
\hline
\end{tabular}

Table S3. RT-qPCR target information

\begin{tabular}{ccccc}
\hline Gene Name & Accession number & Amplicon length & Location & Splice variants \\
\hline HELB & NM_033647 & 125 & Exon 4 & $1-4$, X2 \\
MYC (c-MYC) & NM_002467 & 108 & Exon 1-2 & $1-2$ \\
ACTB (B-actin) & NM_001101 & 148 & Exon 3-4 & $1-2$ \\
\hline
\end{tabular}




\section{Supplemental Figure 1}

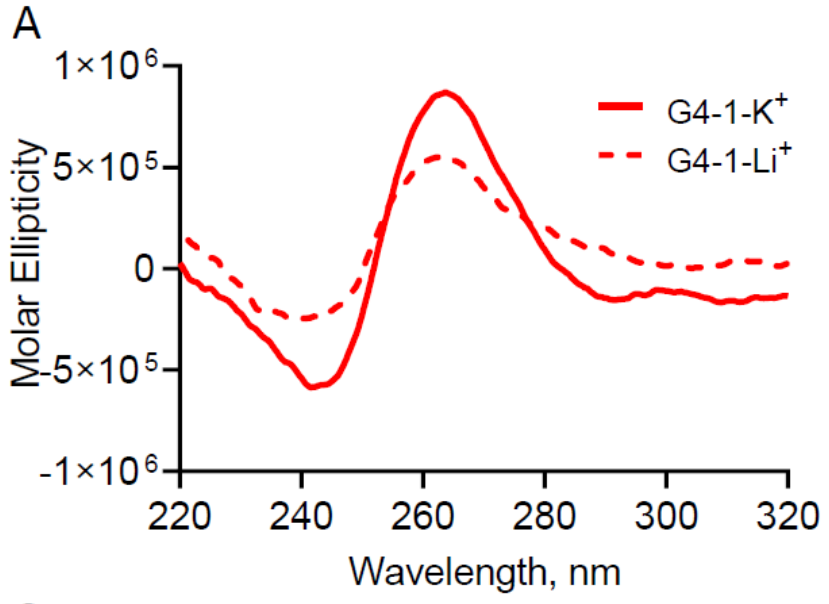

C

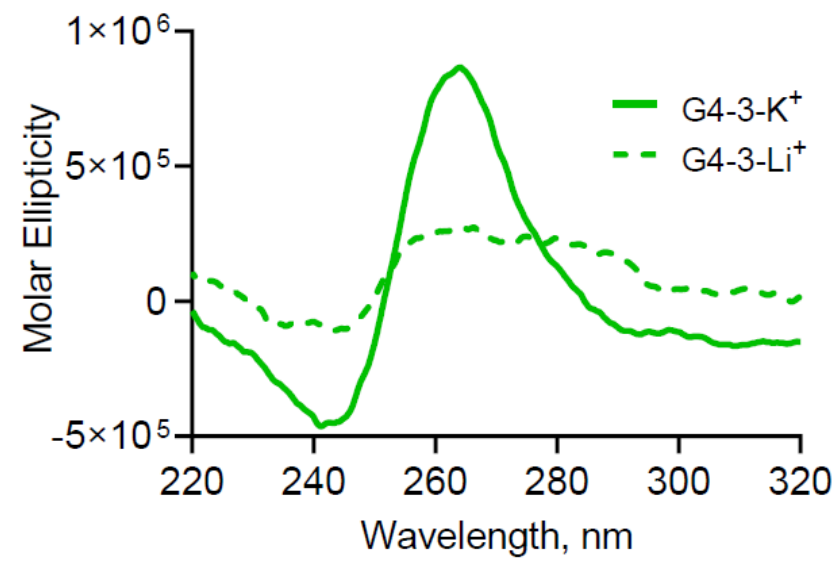

$B$
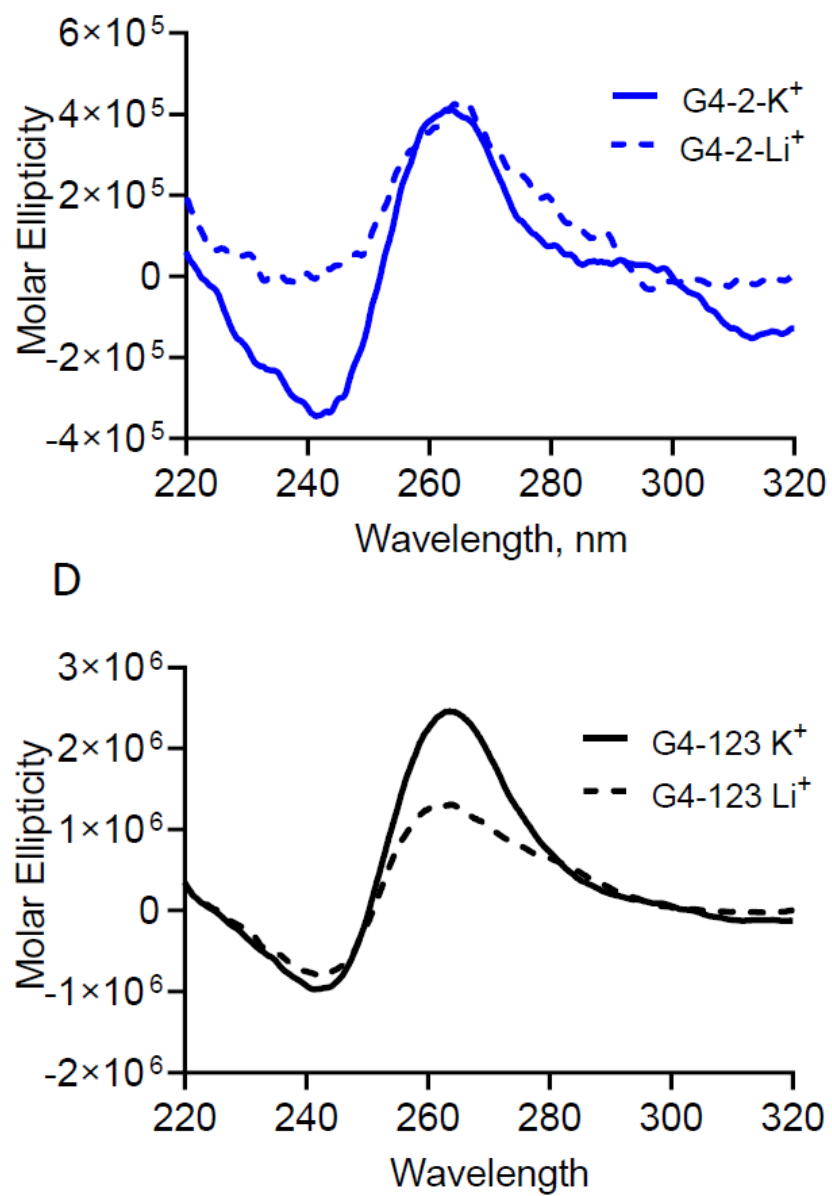

Figure S1. CD spectra of sequences from the HELB promoter are more characteristic of quadruplex structures in $\mathrm{K}^{+}$than $\mathrm{Li}^{+}$. A-C. Circular dichroism spectroscopy of each of the $\mathrm{G} 4$ sequences individually in $\mathrm{K}^{+}$and $\mathrm{Li}^{+}$. D. Circular dichroism spectroscopy of the entire $\mathrm{G}$-rich sequence in $\mathrm{K}^{+}$and $\mathrm{Li}^{+}$. 


\section{Supplemental Figure 2}

A

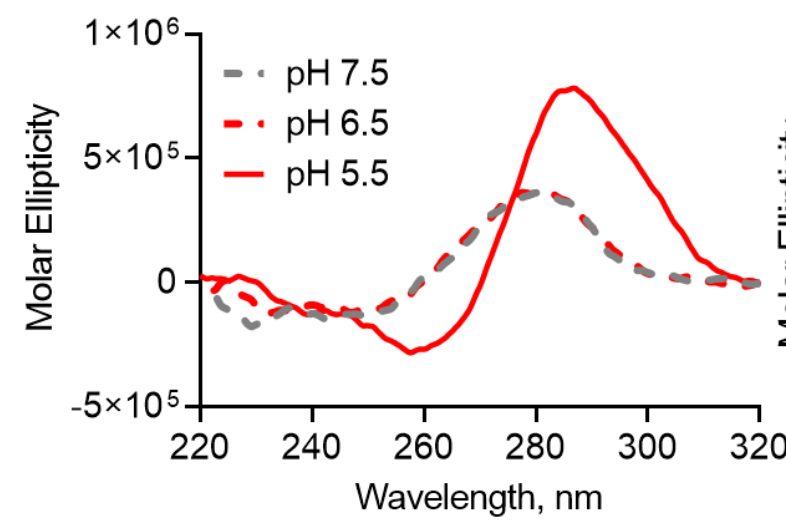

B

iM-2

C

iM-3
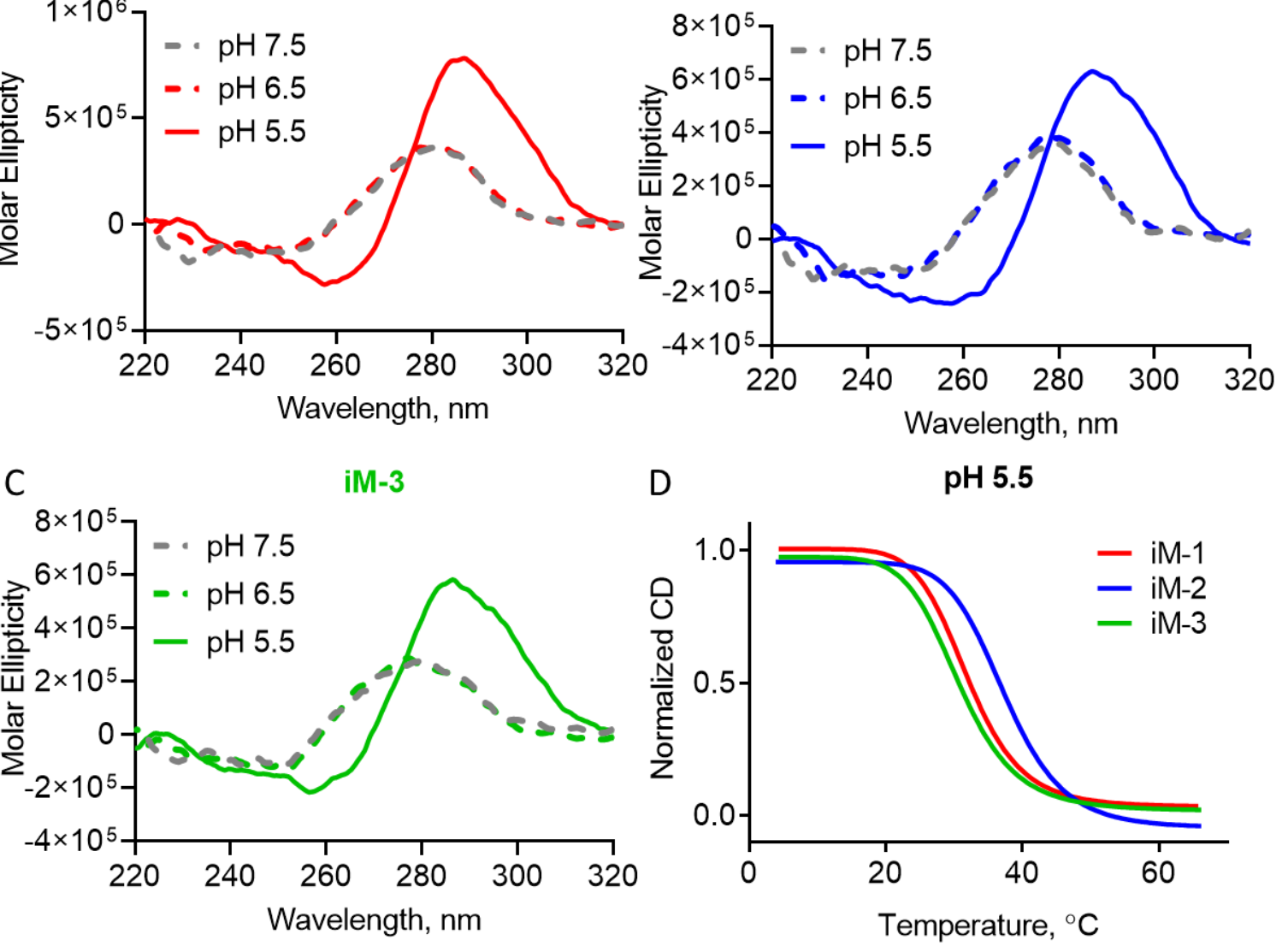

D

pH 5.5

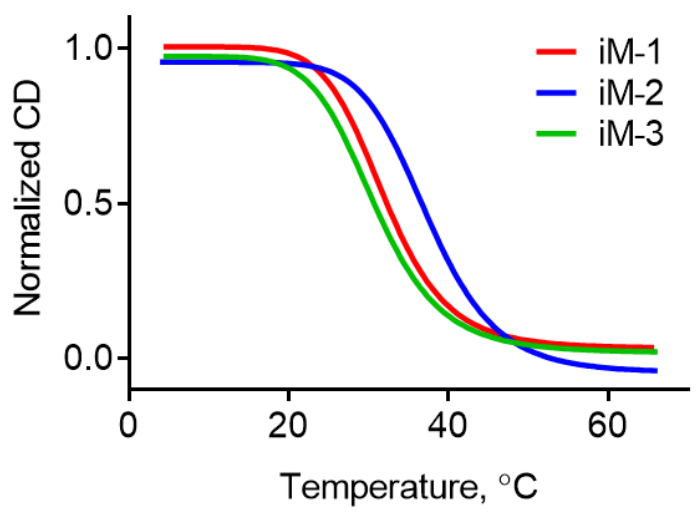

E

iM-123

F
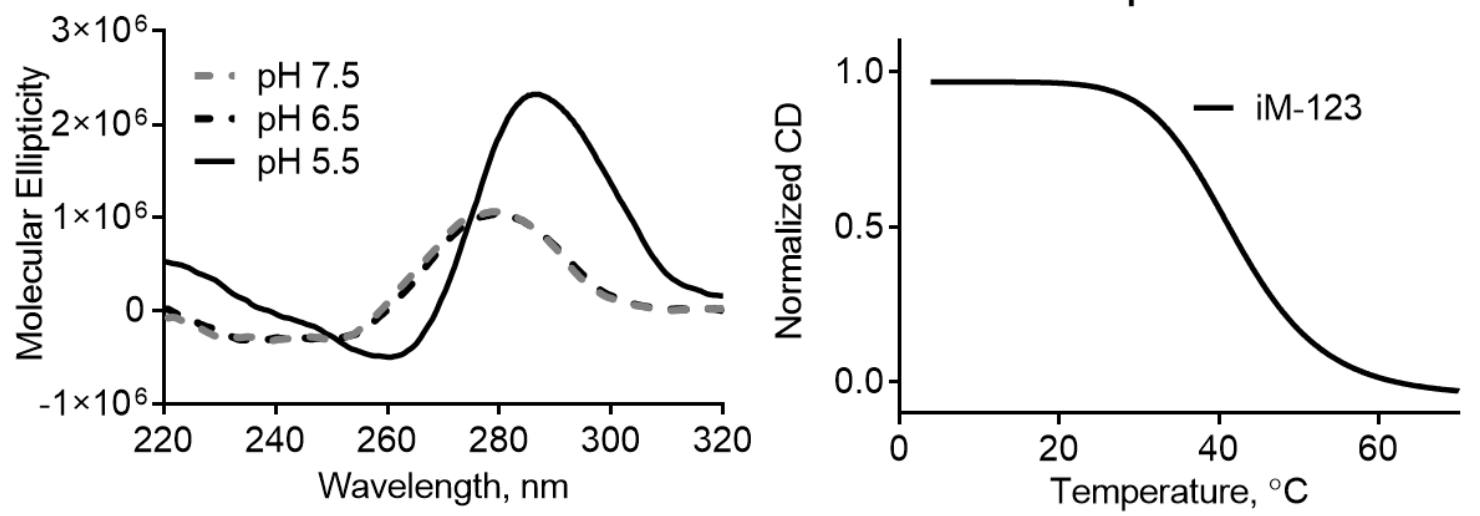

Figure S2. All three of the iM sequences from the HELB promoter fold into iM structures at $\mathrm{pH}$ 5.5. A-C. Circular dichroism spectroscopy of each of the iM sequences individually indicates that they each form $\mathrm{iM}$ structures at $\mathrm{pH} 5.5$ but not at higher $\mathrm{pH}$. D. Melting temperature measurement by $\mathrm{CD}$ indicates that the $\mathrm{T}_{\mathrm{mS}}$ of HELB-iM-1, HELB-iM-2, and HELB-iM-3 at pH 5.5 are $32{ }^{\circ} \mathrm{C}, 37^{\circ} \mathrm{C}$, and $32{ }^{\circ} \mathrm{C}$, respectively. E. CD spectrum of the entire $\mathrm{C}$-rich sequence containing all $3 \mathrm{iM}$ sequences indicates formation of an iM at $\mathrm{pH}$ 5.5. F. The melting temperature of the entire $\mathrm{C}$-rich sequence by $\mathrm{CD}$ at $\mathrm{pH} 5.5$ is $42{ }^{\circ} \mathrm{C}$. 